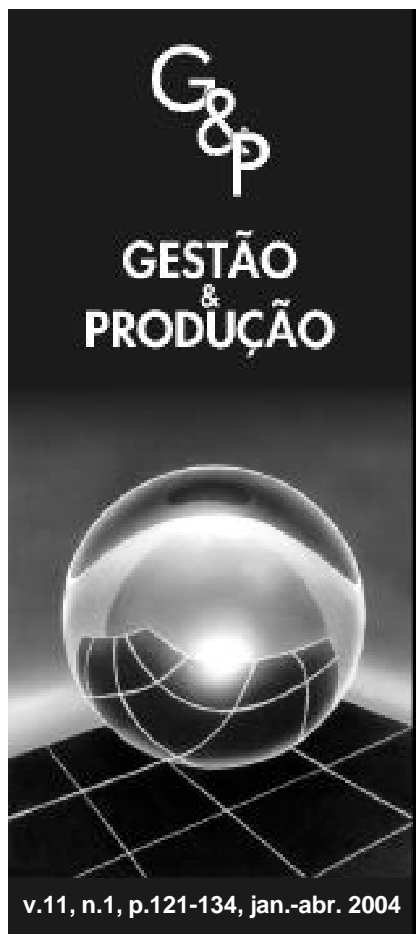

\title{
APLICAÇÃO DAS TÉCNICAS DE PLANEJAMENTO E ANÁLISE DE EXPERIMENTOS NO PROCESSO DE INJEÇÃO PLÁSTICA
}

\author{
Edwin V. Cardoza Galdamez \\ Luiz C. Ribeiro Carpinetti \\ Departamento de Engenharia de Produção, \\ Escola de Engenharia de São Carlos, USP, \\ Avenida Trabalhador Sancarlense, 400, CEP 13566-590, São Carlos, SP, \\ e-mails: cardoza@prod.eesc.usp.br e carpinet@prod.eesc.usp.br
}

\section{Resumo}

Experimentos industriais são realizados pelas empresas com o intuito de melhorar o desempenho dos produtos e os processos de fabricação. Nesse sentido, este trabalho tem por objetivo estudar e aplicar as técnicas de planejamento e análise de experimentos na melhoria da qualidade industrial. Especificamente são aplicadas as técnicas de planejamento Fatorial Fracionado $2^{k-p}$, Metodologia de Superfície de Resposta e Análise de Variância, em um processo de moldagem por injeção plástica. Com essa pesquisa experimental foi possível identificar os níveis ótimos de regulagem e os parâmetros mais importantes da injeção plástica: temperatura da máquina e pressão de injeção. Para finalizar é avaliado o procedimento de implementação das técnicas de experimentação e as dificuldades práticas encontradas na empresa.

Palavras-chave: experimentos industriais, fatoriais fracionados $2^{k-p}$, metodologia de superfície de resposta, processo de moldagem por injeção plástica.

\section{Introdução}

Nos últimos anos, percebe-se que os produtos utilizados e comercializados por diferentes setores da indústria brasileira (construção civil, calçados, brinquedos, agricultura, utilidades domésticas, saúde, embalagens, eletroeletrônicos, aviação e automobilístico) vêm sendo fabricados cada vez mais com diferentes resinas termoplásticas. A evolução do consumo desses materiais é produzida pelo avanço tecnológico dos equipamentos de fabricação, padrões de qualidade, redução do peso dos produtos, facilidade de fabricação, exigências ambientais, consumidores, custos de aplicação e pelas vantagens comerciais que esses produtos representam para a economia nacional (Gazeta Mercantil, 2002). Portanto, nota-se que para tornar esse setor mais competitivo e produtivo é necessário que as empresas busquem reduzir as perdas econômicas decorrentes da má qualidade dos produtos e processos de fabricação.
Com o objetivo de identificar os parâmetros de controle do processo de moldagem plástica por injeção que mais ocasionam problemas de qualidade aos produtos, assim como determinar quais os melhores valores de ajustagem desses parâmetros para minimizar os problemas, o presente trabalho descreve a aplicação e o processo de implementação das técnicas de planejamento e análise de experimentos industriais em uma empresa do setor de plásticos.

O projeto contribui com o processo de divulgação das ferramentas de qualidade no País. Isso é importante porque pesquisas realizadas no Brasil demonstram que tais métodos são pouco difundidos entre as empresas (Kruglianskas, 1995). O trabalho do autor afirma que, apesar do avanço das metodologias de gestão de melhoria da qualidade e mudança empresarial, há deficiências técnicas específicas relacionadas ao desenvolvimento de projetos de produtos e processos de fabricação. Tais deficiências são decorrentes da falta de 
conhecimento estatístico que os gerentes, funcionários e engenheiros das empresas enfrentam ao usar as ferramentas de qualidade.

A seguir são descritas as técnicas de planejamento e análise de experimentos utilizadas no processo de fabricação. Em seguida, serão apresentados os resultados e as análises estatísticas dos experimentos. Para finalizar, são discutidas algumas recomendações e conclusões sobre o processo de implementação dessas ferramentas estatísticas de qualidade.

\section{Técnicas de planejamento e análise de experimentos}

Para Montgomery (1991), as técnicas de planejamento e análise de experimentos (Design of Experiment - DOE) são utilizadas para melhorar as características de qualidade dos produtos e processos de fabricação, reduzir o número de testes e otimizar o uso de recursos da empresa (material, tempo dos funcionários, disponibilidade de equipamentos, etc.).

Com a finalidade de melhorar a qualidade industrial, a produtividade, o desempenho do produto final, os custos das operações, entre outras características, as empresas realizam vários experimentos para encontrar os níveis ótimos dos parâmetros que regulam seus processos de fabricação. Alguns dos problemas encontrados ao realizar os ensaios é a necessidade de estudar simultaneamente o efeito dos fatores com diferentes níveis de regulagens. Nesse caso, observa-se que o número de testes requeridos para a experimentação tende a crescer à medida que a quantidade de fatores aumenta. Isso torna os experimentos industriais inviáveis economicamente, visto que os custos e o tempo de execução são elevados. Antony et al. (1998), Coleman \& Montgomery (1993), Montgomery (1991) e Steinberg \& Hunter (1984) afirmam que tais problemas podem ser contornados quando os experimentos são planejados e analisados com métodos e técnicas estatísticas.

Os objetivos dessas ferramentas são garantir que as informações sejam confiáveis e que os recursos disponíveis para experimentação sejam bem utilizados (Coleman \& Montgomery, 1993; Montgomery, 1991). Um resumo das principais ferramentas é apresentado no Quadro 1. Para outras informações, os autores recomendam consultar a bibliografia específica do tema.

\section{Quadro 1 - Descrição das técnicas de planejamento e análise de experimentos.}

\begin{tabular}{|c|c|c|}
\hline Ferramenta & Características & Bibliografia \\
\hline $\begin{array}{l}\text { Planejamento } \\
\text { fatorial }\end{array}$ & $\begin{array}{l}\text { Utilizada quando todas as combinações dos níveis dos fatores de } \\
\text { controle são realizadas. }\end{array}$ & \multirow{3}{*}{$\begin{array}{l}\text { Montgomery (1991), } \\
\text { Devor et al. (1992), } \\
\text { Chew (1957) }\end{array}$} \\
\hline $\begin{array}{l}\text { Planejamento } \\
\text { fatorial } 2^{\mathrm{k}}\end{array}$ & Técnica com dois níveis e $2^{\mathrm{k}}$ número de combinações de $k$ fatores. & \\
\hline $\begin{array}{l}\text { Planejamento } \\
\text { fatorial } \\
\text { fracionado } 2^{\mathrm{k}-\mathrm{p}}\end{array}$ & $\begin{array}{c}\text { Utilizado quando há vários fatores de controle e não é viável } \\
\text { economicamente para as empresas realizar todas as combinações dos } \\
\text { experimentos. }\end{array}$ & \\
\hline $\begin{array}{l}\text { Metodologia de } \\
\text { superfície de } \\
\text { resposta }\end{array}$ & $\begin{array}{l}\text { Response surface methodology (MSR) é um conjunto de técnicas de } \\
\text { planejamento e análise de experimentos usadas na modelagem } \\
\text { matemática de respostas. Ou seja, procura-se identificar o } \\
\text { relacionamento que existe entre os parâmetros, representados por } \\
\text { variáveis quantitativas, como tempo, velocidade, pressão, temperatura, } \\
\text { etc., e as respostas do sistema analisado. }\end{array}$ & \multirow[t]{2}{*}{$\begin{array}{l}\text { Hill \& Hunter (1966), } \\
\text { Myers \& Montgomery } \\
\quad \text { (1995) }\end{array}$} \\
\hline $\begin{array}{l}\text { Planejamento } \\
\text { fatorial } 2^{\mathrm{k}}, \text { com } \\
\text { pontos centrais } \\
\end{array}$ & $\begin{array}{l}\text { Esse método consiste em adicionar um ponto de experimentação no } \\
\text { nível intermediário aos níveis investigados para os } k \text { fatores de controle. }\end{array}$ & \\
\hline $\begin{array}{l}\text { Análise de } \\
\text { variância }\end{array}$ & $\begin{array}{l}\text { Analysis of variance (ANOVA), é uma ferramenta que permite estudar } \\
\text { se há diferenças significativas entre as respostas experimentais. }\end{array}$ & \multirow{4}{*}{$\begin{array}{l}\text { Montgomery (1991), } \\
\text { Devor et al. (1992) }\end{array}$} \\
\hline \multirow{3}{*}{ Gráficos } & $\begin{array}{l}\text { Os gráficos de efeitos principais ilustram a variação média das } \\
\text { respostas em função da mudança no nível de um fator, mantendo os } \\
\text { outros fatores constantes. }\end{array}$ & \\
\hline & $\begin{array}{l}\text { Os gráficos de efeitos de interação descrevem a variação média de um } \\
\text { fator em função dos níveis de outros fatores. }\end{array}$ & \\
\hline & $\begin{array}{c}\text { O gráfico de probabilidade normal é utilizado nas situações em que não } \\
\text { é possível repetir um experimento e é importante obter uma estimativa } \\
\text { independente do erro experimental para julgar a importância dos efeitos } \\
\text { principais e de interação. }\end{array}$ & \\
\hline
\end{tabular}




\section{Experimentos industriais}

Os conceitos das técnicas de planejamento e análise de experimentos foram aplicados em uma empresa do setor de resinas termoplásticas, localizada no interior do Estado de São Paulo. As principais atividades da empresa são o desenvolvimento, a fabricação, a montagem e a comercialização de produtos de plástico (assentos sanitários, armários e espelhos, caixas d'água, banheiras, acessórios para banheiros, cantoneiras e chuveiros, válvulas e adaptadores e mangueiras).

$\mathrm{Na}$ fase inicial do projeto experimental percebeu-se que já estavam sendo implementadas na empresa algumas ações de melhoria no desenvolvimento de produtos. Dessa forma, a aplicação dos conceitos de projetos experimentais nessa etapa limitava os benefícios que poderiam ser alcançados. No entanto, a companhia vinha enfrentando problemas de qualidade nos processos de fabricação, gerando perdas econômicas e problemas com os clientes (insatisfação, atraso e devolução dos produtos).

Com essas informações, a equipe responsável (pesquisadores, gerentes do controle de qualidade e a engenharia de processos) por conduzir os testes decidiu que a pesquisa se concentraria na engenharia de processos. Ou seja, o principal objetivo do projeto seria melhorar as características de qualidade de um dos processos de fabricação. Especificamente, foi selecionado o processo de moldagem plástica por injeção por ser o mais crítico e com o maior número de problemas de qualidade.

\subsection{Informações técnicas do processo de fabricação}

No processo de moldagem plástica por injeção, os grânulos do polímero são fundidos no cilindro da máquina e, em estado líquido, é injetado no molde por um pistão (Figura 1). Os principais parâmetros de controle são:

- tempo de ciclo: é o tempo necessário para completar o ciclo de injeção;

- velocidade de injeção: é a velocidade de avanço do pistão da máquina injetora;

- tempo de injeção: é o tempo que a máquina utiliza para realizar a operação de injeção e, eventualmente, o recalque da peça injetada;

- tempo de resfriamento: é o tempo que a máquina permanece parada e, ao mesmo tempo, água industrial (gelada) ou água normal circula pelo molde;

- temperatura do molde: fator controlado pela quantidade de água que passa pelos canais de circulação do molde;

- temperatura da máquina: é a temperatura do cilindro da máquina que determina a temperatura do material que será injetado no molde;
- pressão de injeção: é a pressão com que o material é injetado no molde;

- pressão de fechamento: é a pressão utilizada para regular e fechar o molde; e

- pressão de recalque: é a pressão que atua dentro do tempo de recalque. Essa operação é realizada no processo de moldagem plástica para garantir que todas as cavidades do molde sejam completamente preenchidas.

Os principais defeitos que ocorrem nesse processo em razão da variação constante dos parâmetros de controle são: rebarba ou falha, deformação geométrica do componente, erros dimensionais, bolhas de ar, as linhas de encontro do fluxo do polímero aparecem com intensidade e o componente é frágil.

Segundo Cominatto (1997), a falha é decorrente de pouco material injetado no molde. Isso pode ocorrer quando a pressão é insuficiente, o tempo de injeção é pequeno ou o material não está totalmente fundido. Por outro lado, quando muito material é injetado no molde, isto é, o tempo de injeção e a pressão de injeção são elevados, ocorre a rebarba. Os defeitos geométricos de deformação e os erros dimensionais ocorrem quando o material é injetado a uma temperatura elevada e o tempo de resfriamento é pequeno. As bolhas de ar internas aparecem quando a pressão de injeção é baixa e a temperatura do cilindro é alta, porque o molde está frio ou o sistema de canais de alimentação é deficiente. Finalmente, o componente é frágil quando o ciclo de trabalho é irregular, o sistema de alimentação é deficiente ou o material não alcançou a temperatura ideal de trabalho.

Percebe-se que os defeitos que ocorrem nos componentes moldados por injeção podem ter mais de uma causa provável. Uma prática adotada pela empresa para regular o processo e reduzir os defeitos é a construção de uma ficha técnica para cada um dos produtos fabricados. Nesse cadastro são apresentados, com base no conhecimento prático dos funcionários, os valores dos parâmetros que os operários devem utilizar na hora de regular a máquina, durante o funcionamento ou na etapa de preparação (set-up).

Isto é, ao notar os defeitos no processo, o responsável pelo setor da manufatura deve mudar os valores dos fatores de controle, até obter resultados com qualidade satisfatória. Porém, ainda que os operadores da máquina e supervisores tenham grande conhecimento e experiência nos processos de fabricação utilizados pela empresa, eles perdem muito tempo e material para encontrar as regulagens ideais. Portanto, percebe-se que aplicar e apresentar aos funcionários os conceitos das técnicas de planejamento e análise de experimentos é importante, visto que isso poderá reduzir esses problemas e determinar com mais segurança os níveis dos fatores que influenciam o processo de fabricação. 


\subsection{Seleção dos fatores de controle, níveis de ajustagem e variáveis de respostas}

A partir das reuniões, verificou-se que, entre todos os fatores de controle, os que mais afetam a qualidade do resultado do processo são: tempo de injeção, tempo de resfriamento, temperatura do molde, temperatura da máquina, velocidade de injeção e pressão de injeção. Esses fatores foram selecionados porque respondem mais rápido às mudanças que ocorrem nas regulagens do processo e são os mais sensíveis às variações provocadas pelas fontes internas e externas ao processo (Cominatto, 1997). Ainda nos estudos realizados por Acharya \& Mahesh (1999), Engel \& Huele (1996), Taguchi (1993) \& Montgomery (1991), alguns desses fatores foram investigados e considerados como os principais no processo de injeção plástica. A ficha técnica do processo de fabricação e os níveis de ajustagem dos fatores de controle para a primeira rodada de experimentos são apresentados na Tabela 1.

É importante ressaltar que, apesar de o componente plástico ser produzido e comercializado há vários anos, no início desta etapa não existia essa ficha técnica. Assim, para reunir tais informações foi necessário programar junto com a área de planejamento e controle de produção a fabricação do produto.

Nesse caso, os pesquisadores apenas observaram e acompanharam o método que os funcionários utilizam para regular o processo de injeção plástica. Segundo a equipe responsável por acompanhar os experimentos industriais, as variáveis de resposta que deveriam ser analisadas eram: falha ou rebarba, erro dimensional e deformação geométrica dos componentes. Essas respostas foram consideradas porque são as que mais ocorrem e representam as maiores perdas econômicas no processo de fabricação.

No projeto experimental, para avaliar a falha e a rebarba do componente plástico foi construída uma escala variando entre as notas -10 (falha) e +10 (rebarba). Para os problemas de deformação e erro dimensional, a escala varia de 0 a 10 , sendo que a nota 10 é dada quando o produto apresenta deformação ou erro dimensional e 0 quando o componente plástico é aprovado pelo controle de qualidade.

\subsection{Identificação dos principais fatores de controle}

Considerando-se os seis fatores de controle e seus dois níveis de ajustagem, foi construída a matriz de planejamento. Com o objetivo de reduzir o número de fatores de controle e facilitar o planejamento dos experimentos, todas as zonas de temperatura da máquina foram consideradas como um único fator do processo. Observou-se, ainda, que nesse caso era inviável executar o experimento completo, decidindose, por isso, utilizar a técnica de Fatorial Fracionado $2^{\mathrm{k}-\mathrm{p}}$ $(\mathrm{k}=6$ e $\mathrm{p}=2)$, com resolução IV. Com o experimento Fatorial Fracionado $2^{6-2}-$ (Tabela 2), foi possível reduzir o número de experimentos, o tempo de execução e o consumo do material de transformação.

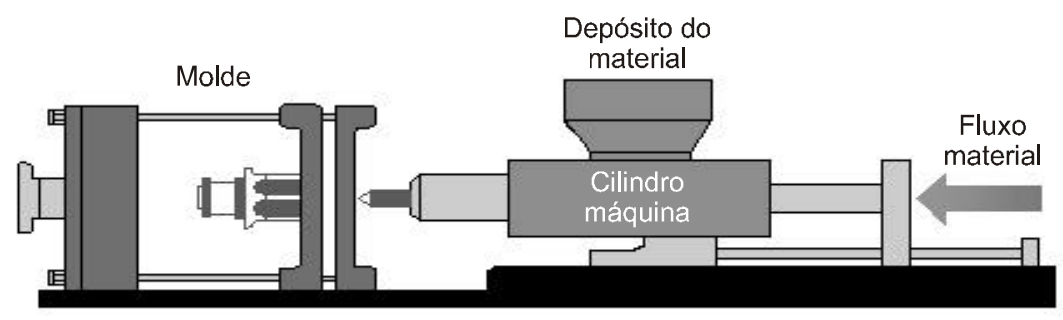

Figura 1 - Processo de moldagem plástica por injeção.

Tabela 1 - Ficha técnica e níveis de ajustagem utilizados no experimento.

\begin{tabular}{|c|c|c|c|c|c|c|}
\hline \multirow{2}{*}{ Fatores } & \multirow{2}{*}{\multicolumn{2}{|c|}{ Ficha técnica }} & \multicolumn{4}{|c|}{ Níveis de ajustagem } \\
\hline & & & \multicolumn{2}{|c|}{-1} & \multicolumn{2}{|c|}{+1} \\
\hline 1. Tempo de injeção & \multicolumn{2}{|c|}{3 segundos (s) } & \multicolumn{2}{|c|}{$2(s)$} & \multicolumn{2}{|c|}{$10(\mathrm{~s})$} \\
\hline 2. Tempo de resfriamento & \multicolumn{2}{|c|}{7 segundos (s) } & \multicolumn{2}{|c|}{$6(\mathrm{~s})$} & \multicolumn{2}{|c|}{$15(\mathrm{~s})$} \\
\hline 3. Temperatura do molde & \multicolumn{2}{|c|}{ água industrial/água normal } & \multicolumn{2}{|c|}{ água industrial } & \multicolumn{2}{|c|}{ água normal } \\
\hline \multirow{2}{*}{ 4. Temperatura da máquina } & $\mathrm{Z}_{1}=190^{\circ} \mathrm{C}$ & $\mathrm{Z}_{3}=175^{\circ} \mathrm{C}$ & $\mathrm{Z}_{1}: 190^{\circ} \mathrm{C}$ & $\mathrm{Z}_{3}: 165^{\circ} \mathrm{C}$ & $\mathrm{Z}_{1}: 190^{\circ} \mathrm{C}$ & $\mathrm{Z}_{3}: 230^{\circ} \mathrm{C}$ \\
\hline & $\mathrm{Z}_{2}=180^{\circ} \mathrm{C}$ & $\mathrm{Z}_{4}=170^{\circ} \mathrm{C}$ & $\mathrm{Z}_{2}: 170^{\circ} \mathrm{C}$ & $\mathrm{Z}_{4}: 160^{\circ} \mathrm{C}$ & $\mathrm{Z}_{2}: 240^{\circ} \mathrm{C}$ & $\mathrm{Z}_{4}: 220^{\circ} \mathrm{C}$ \\
\hline 5. Velocidade de injeção & \multicolumn{2}{|c|}{$50 \%$} & \multicolumn{2}{|c|}{$40 \%$} & \multicolumn{2}{|c|}{$100 \%$} \\
\hline 6. Pressão de injeção & \multicolumn{2}{|c|}{$20 \mathrm{BAR}$} & \multicolumn{2}{|c|}{18 BAR } & \multicolumn{2}{|c|}{$30 \mathrm{BAR}$} \\
\hline
\end{tabular}


Para realizar os ensaios utilizou-se um molde com quatro cavidades e cada uma das combinações dos fatores de controle foi realizada cinco vezes.

Ainda, por conveniência, determinou-se que a seqüência dos testes seria definida pela temperatura da máquina. Isso era necessário porque o tempo de estabilização da temperatura do cilindro é muito grande, fato que inviabilizaria a realização dos experimentos em apenas um dia se fosse necessário alternar o nível desse fator de controle.

\subsubsection{Análise de resultados}

Nesta fase do procedimento experimental, utilizando-se a escala numérica definida anteriormente, as pessoas responsáveis pelo controle de qualidade dos produtos classificaram os componentes plásticos. A média dos resultados é apresentada na Tabela 3. Com os dados dessa tabela são estimados os efeitos principais e de interação, conforme a Tabela 4. Nesta, observa-se que entre os efeitos principais, os mais significativos são $\mathrm{E}_{4}$ e $\mathrm{E}_{6}$. Entre os efeitos de interação, os mais significativos são $\mathrm{E}_{15}, \mathrm{E}_{23}$ e $\mathrm{E}_{46}$, entretanto, estão confundidos. Assim, com esses resultados torna-se difícil determinar qual a combinação mais importante do processo de moldagem plástica por injeção.

A Figura 2 apresenta os gráficos de probabilidade normal do experimento, nos quais observa-se que alguns efeitos principais e de interação encontram-se distantes da reta. Isso indica que esses efeitos são significativamente diferentes de zero. A partir do gráfico de Falha/Rebarba percebe-se que os principais efeitos significativos são: pressão de injeção e temperatura da máquina $\left(\mathrm{E}_{4}\right.$ e $\left.\mathrm{E}_{6}\right)$. Quanto aos outros efeitos, verifica-se que estão distribuídos ao longo de uma reta, portanto, não são significativos no processo de injeção plástica.

No gráfico de Deformação verifica-se que os efeitos de interação dos fatores $\mathrm{E}_{15}, \mathrm{E}_{23}$ e $\mathrm{E}_{46}$ são significativos, porém, estão confundidos e dificilmente pode-se afirmar qual é o mais importante no processo de fabricação.

Por meio do gráfico de Erros Dimensionais, verificase novamente que os efeitos de interação mais significativos são $\mathrm{E}_{15}, \mathrm{E}_{23}$ e $\mathrm{E}_{46}$. Assim como percebe-se que os efeitos principais diferentes de zero são a pressão de injeção e temperatura da máquina $\left(\mathrm{E}_{4} \mathrm{e} \mathrm{E}_{6}\right)$.

Na Figura 3, são ilustradas as variações dos efeitos principais $\mathrm{E}_{1}, \mathrm{E}_{2}, \mathrm{E}_{3}, \mathrm{E}_{4}, \mathrm{E}_{5}$ e $\mathrm{E}_{6}$, em função dos níveis dos fatores. Os gráficos confirmam a hipótese de que os fatores temperatura da máquina e pressão de injeção são os únicos que produzem efeitos significativos nas respostas do processo de injeção plástica. Com o objetivo de verificar se estatisticamente a variação dos resultados experimentais é significativa no processo de injeção, decidiu-se utilizar a ferramenta de Análise de Variância (ANOVA). Os resultados da ANOVA para cada uma das características de qualidade investigadas no experimento são apresentados na Tabela 5.

Tabela 2 - Experimento fatorial fracionado $2^{6-2}$.

\begin{tabular}{|c|c|c|c|c|c|c|c|}
\hline \multirow{3}{*}{$\begin{array}{c}\text { Teste } \\
1\end{array}$} & \multirow{3}{*}{$\begin{array}{c}\begin{array}{c}\text { Tempo } \\
\text { injeção }\end{array} \\
2\end{array}$} & \multirow{3}{*}{$\begin{array}{c}\begin{array}{c}\text { Tempo } \\
\text { resfriamento }\end{array} \\
6\end{array}$} & \multirow{3}{*}{$\begin{array}{c}\begin{array}{c}\text { Temperatura } \\
\text { molde }\end{array} \\
\text { água industrial }\end{array}$} & \multicolumn{2}{|c|}{$\begin{array}{l}\text { Temperatura } \\
\text { máquina }\end{array}$} & \multirow{3}{*}{$\begin{array}{c}\begin{array}{c}\text { Velocidade } \\
\text { injeção }\end{array} \\
40\end{array}$} & \multirow{3}{*}{$\begin{array}{c}\begin{array}{c}\text { Pressão } \\
\text { injeção }\end{array} \\
18\end{array}$} \\
\hline & & & & $\mathrm{Z}_{1}: 190^{\circ} \mathrm{C}$ & $\mathrm{Z}_{3}: 165^{\circ} \mathrm{C}$ & & \\
\hline & & & & $\mathrm{Z}_{2}: 170^{\circ} \mathrm{C}$ & $\mathrm{Z}_{4}: 160^{\circ} \mathrm{C}$ & & \\
\hline 2 & 10 & 6 & & \multicolumn{2}{|c|}{$"$} & 100 & 18 \\
\hline 3 & 2 & 15 & $"$ & \multicolumn{2}{|c|}{$"$} & 100 & 30 \\
\hline 4 & 10 & 15 & $"$ & \multicolumn{2}{|c|}{$"$} & 40 & 30 \\
\hline 5 & 2 & 6 & água normal & \multicolumn{2}{|c|}{$"$} & 100 & 30 \\
\hline 6 & 10 & 6 & $"$ & \multicolumn{2}{|c|}{ " } & 40 & 30 \\
\hline 7 & 2 & 15 & $"$ & \multicolumn{2}{|c|}{$"$} & 40 & 18 \\
\hline 8 & 10 & 15 & $"$ & \multicolumn{2}{|c|}{$"$} & 100 & 18 \\
\hline \multirow{2}{*}{9} & \multirow{2}{*}{2} & \multirow{2}{*}{6} & \multirow{2}{*}{ água industrial } & $\mathrm{Z}_{1}: 190^{\circ} \mathrm{C}$ & $\mathrm{Z}_{3}: 230^{\circ} \mathrm{C}$ & \multirow{2}{*}{40} & \multirow{2}{*}{30} \\
\hline & & & & $\mathrm{Z}_{2}: 240^{\circ} \mathrm{C}$ & $\mathrm{Z}_{4}: 220^{\circ} \mathrm{C}$ & & \\
\hline 10 & 10 & 6 & $"$ & \multicolumn{2}{|c|}{$"$} & 100 & 30 \\
\hline 11 & 2 & 15 & $"$ & \multicolumn{2}{|c|}{$"$} & 100 & 18 \\
\hline 12 & 10 & 15 & $"$ & \multicolumn{2}{|c|}{$"$} & 40 & 18 \\
\hline 13 & 2 & 6 & água normal & \multicolumn{2}{|c|}{$"$} & 100 & 18 \\
\hline 14 & 10 & 6 & $"$ & \multicolumn{2}{|c|}{$"$} & 40 & 18 \\
\hline 15 & 2 & 15 & $"$ & \multicolumn{2}{|c|}{$"$} & 40 & 30 \\
\hline 16 & 10 & 15 & " & \multicolumn{2}{|c|}{$"$} & 100 & 30 \\
\hline
\end{tabular}


A ANOVA para a Falha/Rebarba demonstra com $90 \%$ de confiança que os fatores que influenciam o desempenho do processo são a temperatura da máquina e a pressão de injeção. Ao mesmo tempo, verifica-se que a velocidade de injeção tem efeito significativo médio. Quanto às outras variáveis, não é possível concluir com segurança quais são os efeitos principais. Isso porque os resultados demonstram que há a probabilidade de desprezar os efeitos desses fatores, visto que podem ser significativos no desempenho do processo de fabricação.
A análise para a resposta Erros Dimensionais demonstra que os fatores temperatura da máquina e pressão de injeção são os que mais produzem efeito significativo. Ao mesmo tempo, percebe-se que os outros parâmetros não influenciam o processo de fabricação. Os resultados da ANOVA para a resposta Deformação Geométrica demonstram que a temperatura da máquina tem efeito significativo médio e que a pressão de injeção é o parâmetro mais significativo no processo de injeção plástica. Assim, pela análise conclui-se que os efeitos principais dos outros fatores são desprezíveis.

Tabela 3 - Resultados dos experimentos.

\begin{tabular}{cccc}
\hline Teste & Fal. reb. & Def. & Dim. \\
\hline 1 & -10 & 10 & 10 \\
2 & -10 & 10 & 10 \\
3 & 0,75 & 4,2 & 0,95 \\
4 & -6 & 7 & 6,4 \\
5 & 2 & 1,2 & 1 \\
6 & 0 & 1 & 1 \\
7 & -10 & 10 & 10 \\
8 & -10 & 10 & 10 \\
9 & 8 & 7,6 & 3,25 \\
10 & 7 & 3 & 1 \\
11 & 2,75 & 4,4 & 1 \\
12 & 0,75 & 5 & 1 \\
13 & 5,75 & 5,3 & 3,7 \\
14 & 2 & 1,6 & 1 \\
15 & 6,25 & 3,4 & 1 \\
16 & 8,5 & 3 & 1,9 \\
\hline
\end{tabular}

Tabela 4 - Efeitos principais e de interação.

\begin{tabular}{|c|c|c|c|c|}
\hline & Fatores & Fal. reb. & Def. & Dim. \\
\hline \multirow{5}{*}{ 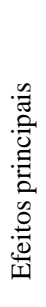 } & $\mathrm{E}_{1}$. Tempo injeção & $-1,66$ & $-0,69$ & 0,18 \\
\hline & $\mathrm{E}_{2}$. Tempo resfriamento & $-1,47$ & 0,91 & 0,16 \\
\hline & $\begin{array}{l}\mathrm{E}_{3} \text {. Temperatura do } \\
\text { molde }\end{array}$ & 1,41 & $-1,96$ & $-0,50$ \\
\hline & $\mathrm{E}_{4}$. T. da máquina & 10,53 & $-2,51$ & $-4,44$ \\
\hline & $\begin{array}{l}\mathrm{E}_{5} . \text { Velocidade de } \\
\text { injeção }\end{array}$ & 1,97 & $-0,56$ & $-0,51$ \\
\hline \multirow{7}{*}{ 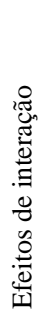 } & $\mathrm{E}_{6}$. Pressão injeção & 6,91 & $-3,24$ & $-3,78$ \\
\hline & $\mathrm{E}_{12}=\mathrm{E}_{35}$ & 0,03 & 1,44 & 1,41 \\
\hline & $\mathrm{E}_{13}=\mathrm{E}_{25}$ & 0,78 & $-0,39$ & $-0,63$ \\
\hline & $\mathrm{E}_{14}=\mathrm{E}_{56}$ & 0,53 & $-1,34$ & $-1,19$ \\
\hline & $\mathrm{E}_{15}=\mathrm{E}_{23}=\mathrm{E}_{46}$ & $-2,28$ & 3,41 & 3,89 \\
\hline & $\mathrm{E}_{16}=\mathrm{E}_{45}$ & $-0,22$ & 0,09 & 0,85 \\
\hline & $\mathrm{E}_{24}=\mathrm{E}_{36}$ & 0,34 & $-1,34$ & $-1,18$ \\
\hline
\end{tabular}



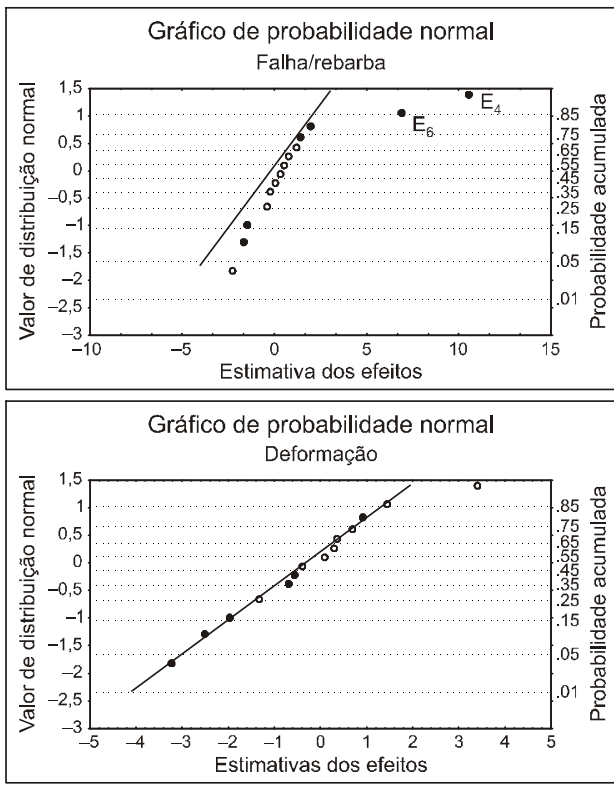

- Efeito de interação

- Efeito principal

- Valor esperado

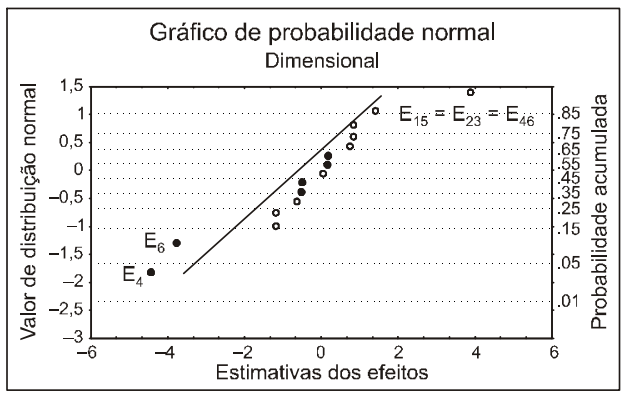

Figura 2 - Gráficos de probabilidade normal.

Depois de realizar a análise de dados, a próxima etapa é apresentar e discutir os resultados com a equipe que acompanha os experimentos. Nessas reuniões, os pesquisadores procuraram demonstrar que o desempenho do processo de injeção plástica é influenciado por apenas dois fatores (temperatura da máquina e pressão de injeção). No entanto, para os funcionários da empresa, essas informações confrontavam-se com as recomendações citadas na ficha técnica do processo. Em razão desse resultado, e com o intuito de validar as conclusões retiradas das análises estatísticas, a equipe decidiu discutir duas propostas, a saber:

1. Um novo experimento poderia ser realizado com o mesmo número de fatores. Porém, adotando-se esta estratégia, a análise estatística poderia levar às mesmas conclusões sobre os parâmetros que influenciam o desempenho do processo de fabricação.

2. A outra proposta era eliminar um dos fatores experimentais e planejar a metade de um experimento completo com cinco fatores, com dois níveis cada um (Fatorial Fracionado $\left.2^{5-1}\right)$. Nesse experimento pode ser utilizada a Resolução V. Com esse critério, os efeitos de interação que ocorrem no processo de injeção plástica serão identificados com mais precisão.

Conforme as estratégias apresentadas e discutidas, a equipe aprovou a segunda proposta. Assim, decidiu-se excluir para a próxima rodada de experimentação o fator velocidade de injeção, visto que a experiência demonstrava que, dentre os fatores considerados no primeiro experimento, este é o último parâmetro que os operadores do processo alteram quando ocorrem problemas de qualidade. A Tabela 6 apresenta os novos níveis dos fatores de controle. Eles foram revistos porque apresentavam uma faixa de variação excessiva e alguns problemas (obstrução do bico de injeção, o cilindro da máquina alcançou temperaturas elevadas e o polímero era deteriorado no cilindro) ocorreram com o primeiro experimento.

Com essas informações foi possível construir um experimento $2^{5-1}$ - Resolução $\mathrm{V}$, com 16 rodadas de teste. Depois de realizar os ensaios, os componentes plásticos foram avaliados pelos funcionários da empresa. Em seguida, foram estimados os efeitos principais e de interação dos fatores de controle e construídos os gráficos de probabilidade normal. 

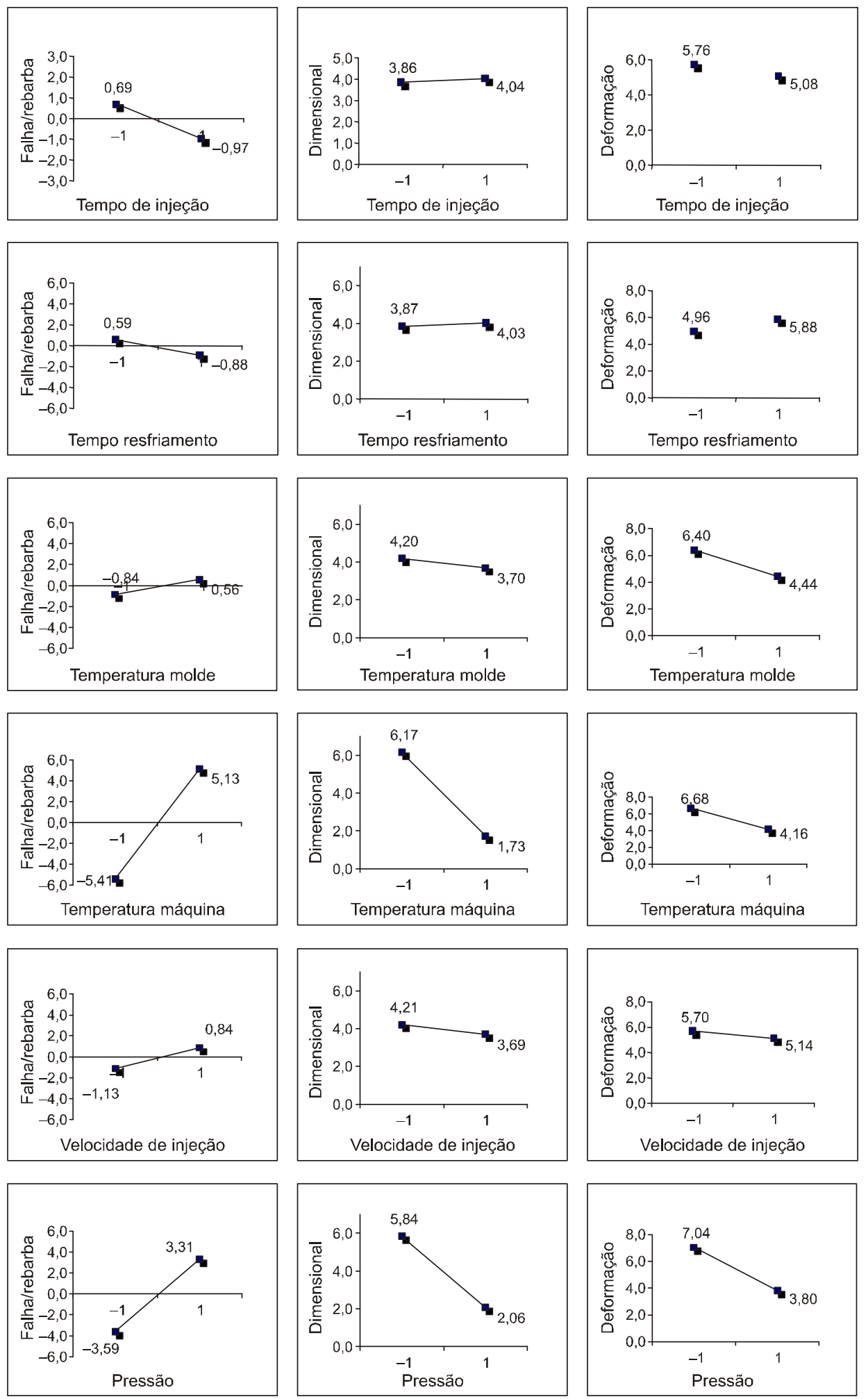

Figura 3 - Gráficos de efeitos principais dos fatores. 
Tabela 5 - Análise de variância $-F_{\alpha=0,1 ; 11: 1 ; v 2: 9}=3,36$.

\begin{tabular}{|c|c|c|c|c|c|c|}
\hline & & & & & & \\
\hline & Fonte de variação & $\begin{array}{c}\text { Soma de } \\
\text { quadrados }\end{array}$ & g.l. & Quadrado médio & $\mathbf{F}$ & Valor $p$ \\
\hline & 1. Tempo injeção & 10,97 & 1 & 10,97 & 3,06 & 0,11 \\
\hline & 2. Tempo resfriamento & 8,63 & 1 & 8,63 & 2,40 & 0,16 \\
\hline & 3. Temperatura molde & 7,91 & 1 & 7,91 & 2,20 & 0,17 \\
\hline & 4. Temperatura máquina & 443,63 & 1 & 443,63 & 123,55 & 1,0 EXP-06 \\
\hline & 5. Velocidade injeção & 15,50 & 1 & 15,50 & 4,32 & 0,07 \\
\hline & 6. Pressão injeção & 190,79 & 1 & 190,79 & 53,13 & 4,6 EXP-05 \\
\hline \multirow[t]{8}{*}{ Falha/rebarba } & Erro $(\varepsilon)$ & 32,32 & 9 & 3,59 & & \\
\hline & Fonte de variação & $\begin{array}{c}\text { Soma de } \\
\text { quadrados }\end{array}$ & g.l. & Quadrado médio & $\mathbf{F}$ & Valor $p$ \\
\hline & 1. Tempo injeção & 0,12 & 1 & 0,12 & 0,01 & 0,91 \\
\hline & 2. Tempo resfriamento & 0,11 & 1 & 0,11 & 0,01 & 0,92 \\
\hline & 3. Temperatura molde & 1,00 & 1 & 1,00 & 0,10 & 0,76 \\
\hline & 4. Temperatura máquina & 78,77 & 1 & 78,77 & 7,96 & 0,02 \\
\hline & 5. Velocidade injeção & 1,05 & 1 & 1,05 & 0,11 & 0,75 \\
\hline & 6. Pressão injeção & 57,00 & 1 & 57,00 & 5,76 & 0,04 \\
\hline \multirow[t]{8}{*}{ Erros dimensionais } & Erro $(\varepsilon)$ & 89,04 & 9 & 9,89 & & \\
\hline & Fonte de variação & $\begin{array}{c}\text { Soma de } \\
\text { quadrados }\end{array}$ & g.l. & Quadrado médio & $\mathbf{F}$ & Valor $p$ \\
\hline & 1. Tempo injeção & 1,89 & 1 & 1,89 & 0,23 & 0,64 \\
\hline & 2. Tempo resfriamento & 3,33 & 1 & 3,33 & 0,41 & 0,54 \\
\hline & 3. Temperatura molde & 15,41 & 1 & 15,41 & 1,91 & 0,20 \\
\hline & 4. Temperatura máquina & 25,25 & 1 & 25,25 & 3,13 & 0,11 \\
\hline & 5. Velocidade injeção & 1,27 & 1 & 1,27 & 0,16 & 0,70 \\
\hline & 6. Pressão injeção & 41,93 & 1 & 41,93 & 5,20 & 0,05 \\
\hline Deformação geométrica & Erro $(\varepsilon)$ & 72,54 & 9 & 8,06 & & \\
\hline
\end{tabular}

Tabela 6 - Novos níveis dos fatores de controle.

\begin{tabular}{ccccccc}
\hline Nível & $\begin{array}{c}\text { Tempo } \\
\text { injeção }\end{array}$ & $\begin{array}{c}\text { Tempo } \\
\text { resfriamento }\end{array}$ & $\begin{array}{c}\text { Temperatura } \\
\text { molde }\end{array}$ & Temperatura máquina & $\begin{array}{c}\text { Pressão } \\
\text { injeção }\end{array}$ \\
\hline \multirow{2}{*}{1} & $2(\mathrm{~s})$ & $6(\mathrm{~s})$ & água industrial & $\mathrm{Z}_{1}: 190^{\circ} \mathrm{C}$ & $\mathrm{Z}_{3}: 165^{\circ} \mathrm{C}$ & $18 \mathrm{BAR}$ \\
& & & & $\mathrm{Z}_{2}: 170^{\circ} \mathrm{C}$ & $\mathrm{Z}_{4}: 160^{\circ} \mathrm{C}$ & \\
+1 & $5(\mathrm{~s})$ & $10(\mathrm{~s})$ & água normal & $\mathrm{Z}_{1}: 190^{\circ} \mathrm{C}$ & $\mathrm{Z}_{3}: 210^{\circ} \mathrm{C}$ & $26 \mathrm{BAR}$ \\
\hline
\end{tabular}

As análises estatísticas (ANOVA) realizadas neste experimento demonstraram que os efeitos principais e de interação provocados pelos parâmetros tempo de injeção, tempo de resfriamento e temperatura do molde não influenciam as características de qualidade. Por outro lado, os testes indicaram e confirmaram que os efeitos principais e de interação mais importantes são produzidos pelos fatores temperatura da máquina e pressão de injeção. Para ver os detalhes desse experimento recomenda-se consultar Galdamez (2002).

\subsection{Otimização do processo de injeção plástica}

O objetivo principal desta etapa do projeto experimental é determinar as condições ótimas do processo de moldagem plástica por injeção. Nessa fase, inicialmente, os pesquisadores apresentaram aos funcionários os conceitos da Metodologia de Superfície de Resposta (Methodology Response Surface - MSR). 
Durante a fase de tomada de decisões, os pesquisadores e os funcionários perceberam que para ajustar o processo de fabricação todas as características de qualidade (Falha/ Rebarba, Deformação Geométrica e Erros Dimensionais) deveriam ser estudadas simultaneamente. O problema de obter a melhor combinação dos fatores para um conjunto de características de qualidade foi resolvido com a construção de uma função resposta, denominada de Soma Quadrática de Respostas - SQ (Taguchi, 1993). A SQ é uma combinação dos valores da escala para as três variáveis de interesse, apresentada pela equação (1).

$$
\begin{aligned}
\mathrm{Y}_{\mathrm{i}}= & (\text { Falha/Rebarba })^{2}+(\text { Deformação })^{2}+\ldots \\
& +(\text { Dimensional })^{2}
\end{aligned}
$$

Com essa função, além de facilitar a interpretação da análise estatística porque todos os valores da resposta serão positivos, será possível observar com mais eficiência as mudanças provocadas por uma das características de qualidade investigadas.

Para construir a matriz experimental foi utilizada a técnica de planejamento fatorial composto central (Myers \& Montgomery, 1995; Montgomery, 1991; Yonchev, 1988). Essa técnica consiste em acrescentar pontos centrais $\left(\mathrm{X}_{0}\right)$ e pontos axiais $( \pm \alpha)$ ao experimento fatorial completo $2^{2}$, conforme apresentado pela Tabela 7 . Nessa matriz de planejamento são apresentados os níveis de ajustagem dos fatores de controle em termos de variáveis naturais e codificadas. Ainda neste experimento, utilizou-se o mesmo molde do primeiro experimento e cada uma das combinações dos fatores de controle foi realizada cinco vezes. Os resultados do experimento são apresentados pela SQ.

\subsubsection{Análise de resultados}

Com os resultados desse experimento e aplicando o método de mínimos quadrados, foi construído um polinômio de primeira ordem (Montgomery, 1991). A ANOVA demonstrou que os dois fatores apresentam efeitos principais e de interação significativos no modelo. Confirmou ainda a existência de uma curvatura no plano da superfície de resposta, o que tornou evidente que a função de aproximação não é linear. Com esses resultados, os pesquisadores concluíram que o modelo de primeira ordem não satisfaz as condições de operação das três variáveis de interesse e, nesse caso, era necessário construir um novo polinômio de ordem superior. O novo modelo matemático é representado pela equação (2).

$$
\begin{aligned}
\mathrm{y}= & 12,35-8,45 \mathrm{x}_{1}-10,14 \mathrm{x}_{2}+20,8 \mathrm{x}^{2}{ }_{1}+15,2 \mathrm{x}_{2}{ }_{2}+\ldots \\
& +42,80 \mathrm{x}_{1} \mathrm{x}_{2}
\end{aligned}
$$

sendo que $\mathrm{x}_{1}$ é a temperatura da máquina e $\mathrm{x}_{2}$, a pressão de injeção.

$\mathrm{Na}$ Tabela 8 é apresentada a análise de variância para esse modelo matemático. $\mathrm{O}$ teste $\mathrm{F}$, com nível de signi- ficância de 1\%, aponta que a falta de ajuste do modelo não é significativa na superfície de resposta. Ainda pelos resultados, pode-se afirmar que as variáveis independentes ou de regressão tem efeito significativo na reposta. Portanto, a análise demonstra que o polinômio de segunda ordem se aproxima das condições ótimas ou reais de operação do processo de injeção plástica.

Antes de determinar os melhores níveis de regulagem do processo será realizada uma análise gráfica da superfície de resposta. Na Figura 4 são apresentados os gráficos dessa superfície (tridimensional e bidimensional), gerados a partir do modelo ajustado. Ao examiná-los, observa-se que as combinações dos parâmetros nos níveis máximos ou mínimos influenciam fortemente o resultado da função reposta. Por outro lado, pela forma geométrica da superfície no intervalo $0-25$, percebe-se que há alguns pontos próximos às condições ótimas que minimizam a função reposta.

Essas características dos gráficos levam a descartar a hipótese de que no espaço formado pelos eixos $\left(\mathrm{x}_{1}, \mathrm{x}_{2}\right)$ apenas um ponto minimiza a função resposta, mas determinam que uma análise mais detalhada dos pontos próximos ao intervalo de resposta $(0-25)$ em todas as direções é necessária. O método utilizado para resolver o problema é conhecido como Análise Canônica de Superfície de Resposta (Myers \& Montgomery, 1995; Montgomery, 1991; Box \& Draper, 1987; Box \& Hunter, 1957; Cochran \& Cox, 1957). O primeiro passo é transformar o modelo ajustado em um novo sistema de coordenadas $\left(\mathrm{w}_{1} \mathrm{e} \mathrm{w}_{2}\right) \mathrm{com}$ a origem no ponto estacionário. A forma canônica do modelo é definida pela equação (3).

$$
\mathrm{y}=10,93-3,89 \mathrm{w}_{1}^{2}+40,41 \mathrm{w}_{2}^{2}
$$

sendo que $\mathrm{w}_{1}$ e $\mathrm{w}_{2}$ representam as variáveis independentes transformadas.

Na equação (3), observa-se que os sinais das raízes são opostos. Isto demonstra que o ponto estacionário é um ponto de sela, conforme é descrito pela Figura 4. Ainda comparando os valores das raízes, percebe-se que a pendente da superfície de resposta na direção $\mathrm{w}_{1}$ tende a ser mais atenuada. Além disso, nos gráficos de superfície de resposta nota-se que, quando o processo é regulado a partir de um ponto na direção $\mathrm{w}_{2}$, as características de qualidade tendem a piorar. Assim, por conveniência, será considerado nulo o deslocamento na direção $\mathrm{w}_{2}$.

A resolução do modelo canônico para uma função resposta otimizada demonstra que as raízes na direção $\mathrm{w}_{1}$ são iguais a $\pm 1,66$. Utilizando as equações matemáticas (equação (4)) que descrevem o relacionamento entre as variáveis normais (x) e as variáveis modificadas (w), é determinada a faixa de valores que minimizam a função resposta, conforme a Tabela 9 .

$\left[\mathrm{w}_{1}=-0,664 \mathrm{x}_{1}+0,746 \mathrm{x}_{2}+0,27\right] \mathrm{e}$

$\left[\mathrm{w}_{2}=0,747 \mathrm{x}_{1}+0,664 \mathrm{x}_{2}-0,21\right]$ 
Tabela 7 - Planejamento fatorial composto central.

\begin{tabular}{|c|c|c|c|c|c|c|}
\hline \multirow{2}{*}{ Teste } & \multicolumn{2}{|c|}{ Variáveis codificadas } & \multicolumn{3}{|c|}{ Variáveis naturais } & \multirow{2}{*}{ SQ } \\
\hline & $\mathbf{x}_{1}$ & $\mathbf{x}_{2}$ & \multicolumn{2}{|c|}{ T. máquina $\left(\mathrm{x}_{1}\right)$} & P. injeção $\left(x_{2}\right)$ & \\
\hline \multirow{2}{*}{1} & \multirow{2}{*}{-1} & \multirow{2}{*}{-1} & $\mathrm{Z}_{1}: 190^{\circ} \mathrm{C}$ & $\mathrm{Z}_{3}: 165^{\circ} \mathrm{C}$ & \multirow{2}{*}{18} & \multirow{2}{*}{135,56} \\
\hline & & & $\mathrm{Z}_{2}: 170^{\circ} \mathrm{C}$ & $\mathrm{Z}_{4}: 160^{\circ} \mathrm{C}$ & & \\
\hline 2 & -1 & +1 & & & 26 & 19,03 \\
\hline \multirow{2}{*}{3} & \multirow{2}{*}{+1} & \multirow{2}{*}{-1} & $\mathrm{Z}_{1}: 190^{\circ} \mathrm{C}$ & $\mathrm{Z}_{3}: 210^{\circ} \mathrm{C}$ & \multirow{2}{*}{18} & \multirow{2}{*}{11,07} \\
\hline & & & $\mathrm{Z}_{2}: 220^{\circ} \mathrm{C}$ & $\mathrm{Z}_{4}: 200^{\circ} \mathrm{C}$ & & \\
\hline 4 & +1 & +1 & & & 26 & 65,75 \\
\hline \multirow{2}{*}{5} & \multirow{2}{*}{0} & \multirow{2}{*}{0} & $\mathrm{Z}_{1}: 190^{\circ} \mathrm{C}$ & $\mathrm{Z}_{3}: 187^{\circ} \mathrm{C}$ & \multirow{2}{*}{22} & \multirow{2}{*}{2,56} \\
\hline & & & $\mathrm{Z}_{2}: 195^{\circ} \mathrm{C}$ & $\mathrm{Z}_{4}: 180^{\circ} \mathrm{C}$ & & \\
\hline 6 & 0 & 0 & & & $"$ & 16,69 \\
\hline 7 & 0 & 0 & & & $"$ & 17,13 \\
\hline 8 & 0 & 0 & & & $"$ & 18,88 \\
\hline 9 & 0 & 0 & & & $"$ & 6,50 \\
\hline \multirow{2}{*}{10} & \multirow{2}{*}{+1.414} & \multirow{2}{*}{0} & $\mathrm{Z}_{1}: 190^{\circ} \mathrm{C}$ & $\mathrm{Z}_{3}: 220^{\circ} \mathrm{C}$ & \multirow{2}{*}{22} & \multirow{2}{*}{48,38} \\
\hline & & & $\mathrm{Z}_{2}: 230^{\circ} \mathrm{C}$ & $\mathrm{Z}_{4}: 210^{\circ} \mathrm{C}$ & & \\
\hline \multirow{2}{*}{11} & \multirow{2}{*}{-1.414} & \multirow{2}{*}{0} & $\mathrm{Z}_{1}: 190^{\circ} \mathrm{C}$ & $\mathrm{Z}_{3}: 155^{\circ} \mathrm{C}$ & \multirow{2}{*}{22} & \multirow{2}{*}{41,21} \\
\hline & & & $\mathrm{Z}_{2}: 160^{\circ} \mathrm{C}$ & $\mathrm{Z}_{4}: 150^{\circ} \mathrm{C}$ & & \\
\hline \multirow{2}{*}{12} & \multirow{2}{*}{0} & \multirow{2}{*}{+1.414} & $\mathrm{Z}_{1}: 190^{\circ} \mathrm{C}$ & $\mathrm{Z}_{3}: 187^{\circ} \mathrm{C}$ & \multirow{2}{*}{27} & \multirow{2}{*}{27,88} \\
\hline & & & $\mathrm{Z}_{2}: 195^{\circ} \mathrm{C}$ & $\mathrm{Z}_{4}: 180^{\circ} \mathrm{C}$ & & \\
\hline 13 & 0 & -1.414 & & & 16 & 41,55 \\
\hline
\end{tabular}

Tabela 8 - Análise de variância, $F_{\alpha=0,01 ; v 1: 3 ; ~ v 2: 4}=16,69$.

\begin{tabular}{lcccc}
\hline \multicolumn{1}{c}{ Fonte de variação } & $\begin{array}{c}\text { Soma de } \\
\text { quadrados }\end{array}$ & g.l. & $\begin{array}{c}\text { Quadrado } \\
\text { médio }\end{array}$ & F \\
\hline Regressão & 12921,70 & 5 & 2584,34 & $8,75^{\text {a }}$ \\
Erro & 2067,68 & 7 & 295,38 & \\
Falta de ajuste do modelo & 1850,32 & 3 & 616,77 & 11,35 \\
Erro quadrático puro & 217,36 & 4 & 54,34 & \\
Total & 14989,39 & 12 & & \\
\hline
\end{tabular}

${ }^{a}$ Significativo no nível de $1 \%-\mathrm{F}_{\alpha=0.01: v 1: 5 ; \text { v2:7 }}=7,46$.

Tabela 9 - Níveis ótimos do processo de fabricação.

\begin{tabular}{|c|c|c|c|}
\hline & & Valores codificados & \\
\hline & $(-0,76,1,16)$ & $(0,32,-0,16)$ & $(1,4,-1,3)$ \\
\hline \multirow{2}{*}{$\begin{array}{l}\text { Temperatura da } \\
\text { máquina }\end{array}$} & $\mathrm{Z}_{1}: 190^{\circ} \mathrm{C} \quad \mathrm{Z}_{3}: 168^{\circ} \mathrm{C}$ & $\mathrm{Z}_{1}: 190^{\circ} \mathrm{C} \quad \mathrm{Z}_{3}: 195^{\circ} \mathrm{C}$ & $\mathrm{Z}_{1}: 190^{\circ} \mathrm{C} \quad \mathrm{Z}_{3}: 223^{\circ} \mathrm{C}$ \\
\hline & $\mathrm{Z}_{2}: 176^{\circ} \mathrm{C} \quad \mathrm{Z}_{4}: 161^{\circ} \mathrm{C}$ & $\mathrm{Z}_{2}: 203^{\circ} \mathrm{C} \quad \mathrm{Z}_{4}: 188^{\circ} \mathrm{C}$ & $\mathrm{Z}_{2}: 231^{\circ} \mathrm{C} \quad \mathrm{Z}_{4}: 216^{\circ} \mathrm{C}$ \\
\hline Pressão de injeção & 24 BAR & $21 \mathrm{BAR}$ & $16 \mathrm{BAR}$ \\
\hline
\end{tabular}

Tabela 10 - Confirmação do ponto ótimo.

\begin{tabular}{|c|c|c|c|c|c|}
\hline \multirow{2}{*}{$\begin{array}{l}\text { Temperatura da } \\
\text { máquina }\end{array}$} & \multirow{2}{*}{$\begin{array}{l}\text { Pressão de } \\
\text { injeção }\end{array}$} & \multicolumn{3}{|c|}{ Resultado } & \multirow{2}{*}{ SQ } \\
\hline & & Fal./Reb. & Def. & Dim. & \\
\hline $\mathrm{Z}_{1}: 190^{\circ} \mathrm{C} \quad \mathrm{Z}_{3}: 195^{\circ} \mathrm{C}$ & & 075 & 06 & 045 & 12 \\
\hline $\mathrm{Z}_{2}: 203^{\circ} \mathrm{C} \quad \mathrm{Z}_{4}: 188^{\circ} \mathrm{C}$ & $21 \mathrm{BAK}$ & $0, / 3$ & 0,0 & 0,43 & 1,2 \\
\hline
\end{tabular}



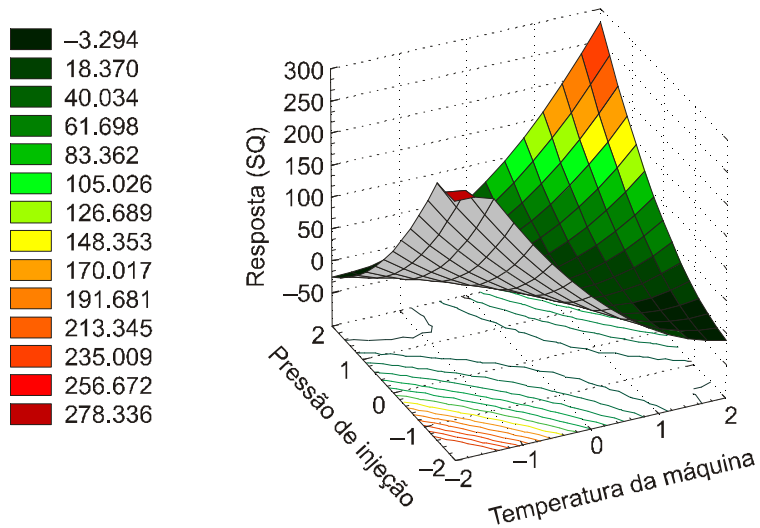

Superfície de resposta tridimensional

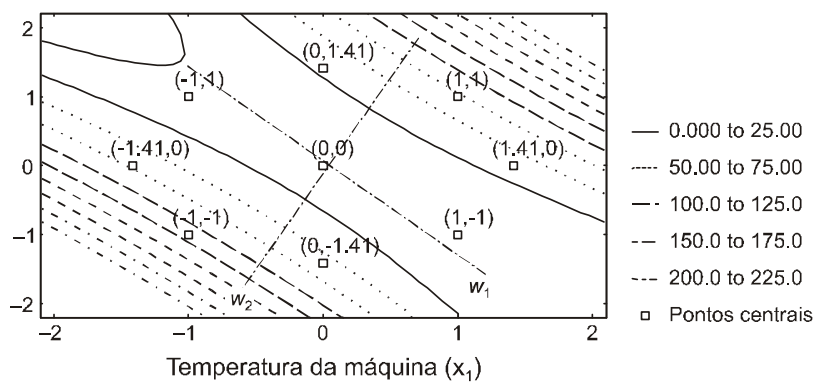

Superfície de contornos bidimensionais

Figura 4 - Gráficos de superfície de resposta.

Nesta fase final dos ensaios, seria necessário realizar um experimento de confirmação dos resultados. Porém, considerando-se as normas de segurança que a empresa define para regular o processo: "...evitar utilizar níveis elevados de temperatura da máquina porque reduzem a vida útil do cilindro de deslocamento e alteram as propriedades químicas do material... por outro lado, as temperaturas baixas não conseguem aquecer o material e conseqüentemente $o$ atrito nas paredes do cilindro maior...", recomenda-se que os níveis máximo e mínimo da faixa de variação devem ser evitados na regulagem do processo de fabricação. Para validar o ponto ótimo, foram realizados testes apenas com o ponto intermediário e os resultados são apresentados na Tabela 10.

Nesse experimento, os outros fatores investigados no projeto continuaram sendo regulados com os mesmos níveis que a empresa determinou na primeira etapa do procedimento experimental. Assim, comparando-se o resultado da função quadrática (SQ) com o resultado do modelo ajustado (variação próxima de 11), a equipe responsável por conduzir os experimentos confirma que tais níveis podem ser definidos como as condições ótimas do processo de moldagem plástica por injeção.

\section{Conclusão}

Primeiramente, com o projeto, pôde-se mostrar aos responsáveis pela engenharia do processo que, diferentemente do que eles afirmavam, nem todos os fatores de ajustagem inicialmente considerados afetam a qualidade do resultado do processo de fabricação. Ou seja, para as faixas de ajustagem consideradas, apenas dois fatores apresentaram efeito significativo sobre as características de qualidade do componente. Para os outros fatores, pode-se afirmar que não há necessidade de definir um valor específico de ajustagem da máquina, mas, sim, uma faixa de valores, dentro da qual o processo terá bom desempenho.

Com a pesquisa experimental, foi possível apresentar uma metodologia de planejamento de experimentos industriais aos funcionários de uma empresa. Esse aspecto certamente motiva as pessoas a desenvolverem os projetos de produtos ou processos de fabricação com mais eficiência e eficácia. O procedimento de otimização utilizado para estabelecer as condições ótimas do processo de injeção plástica foi bastante explorado e descrito para os funcionários. Os resultados motivaram as pessoas a utilizarem as ferramentas estatísticas a fim de melhorar a qualidade de outros produtos e processos de fabricação. Além 
disso, foi possível desenvolver uma pesquisa em que várias características de qualidade são analisadas simultaneamente. Isso é importante porque a maioria dos estudos publicados na literatura de experimentos industriais analisa as respostas individualmente.

Ao longo da pesquisa experimental também foram verificadas algumas dificuldades. A principal está relacionada à escolha dos fatores de controle investigados com as técnicas experimentais. Algumas pesquisas realizadas com o mesmo processo de injeção plástica demonstraram que outros fatores podem influenciar o desempenho.

Deve-se observar, ainda, que, na fase inicial de experimentação, a faixa de operação dos parâmetros foi estabelecida conforme a experiência dos funcionários. Quando os ensaios foram realizados com esses níveis, vários problemas ocorreram com o equipamento de injeção. Considerando esse ponto e outros aspectos (falta de experiência com as técnicas de planejamento e análise de experimentos e de conhecimento do processo de injeção plástica), os pesquisadores, com o primeiro experimento, não conseguiram descobrir com segurança quais fatores realmente influenciavam as características de qualidade. Entretanto, pode-se perceber que, pela falta de procedimentos e técnicas adequadas na definição dos níveis, o processo de fabricação estava perdendo produtividade, gerando refugo e baixa qualidade e aumentando os custos de produção. Essas questões demonstram a necessidade de utilizar as ferramentas estatísticas e realizar novos experimentos. Finalmente, um resultado bastante importante deste trabalho foi o de mostrar às pessoas envolvidas no estudo a validade de analisar cientificamente o processo de transformação e, com isso, não só fomentar o uso dessas técnicas na empresa em questão, mas também promover a aproximação universidade e empresa.

\section{Referências Bibliográficas}

ACHARYA, U. H.; MAHESH, C. Winning back the customer's confidence: a case study on the application of design of experiments to and injection-molding process. Quality Engineering, v. 3, n. 11, p. 357-363, 1999.

ANTONY, J.; KATE, M.; FRANGOU, A. A strategic methodology to the use of advanced statistical quality improvement techniques. The TQM Magazine, v. 10, n. 3, p. 169-176, 1998.

BOX, G. E. P.; DRAPER, N. R. Empirical model-building and response surfaces. New York: Wiley, 1987. (Wiley Series in Probability and Mathematical Statistics).

BOX, G. E. P.; HUNTER, J. S. Experimental design for the exploration and exploitation of response surfaces. In: CHEW, V. Experimental design in industry. New York: John Wiley \& Sons, 1957. p. 138-190.

CHEW, V. Experimental designs industry. New York: John Wiley \& Sons, 1957. p. 1-58.

COCHRAN, W. G.; COX, G. M. Experimental designs. 2. ed. London: John Wiley, 1957.

COLEMAN, D. E.; MONTGOMERY, D. C. A systematic approach to planning for a designed industrial experiment. Technometrics, v. 35, n. 1, p. 1-12, Feb. 1993.

COMINATTO, A. C. Influências das variáveis do ciclo no produto final. São Paulo: ASTRA S/A Indústria e Comércio, 1997. (Apostila).

DEVOR, R. E.; CHANG, T.; SUTHERLAND, J. W. Statistical quality design and control-Contemporary concepts and methods. New Jersey: Prentice Hall, 1992. p. 503-744.

ENGEL, J.; HUELE, A. F. A generalized linear modeling approach to robust design. Technometrics, v. 38, n. 4, p. 365-373, 1996.
GALDAMEZ, E. V. C. Aplicação das técnicas de planejamento $e$ análise de experimentos na melhoria da qualidade industrial de um processo de fabricação de produtos plásticos. 2002. 133p. Dissertação (Mestrado) - Escola de Engenharia de São Carlos, São Carlos.

HILL, W. J.; HUNTER, W. G. A review of response surface methodology: a literature survey. Technometrics, v. $8, \mathrm{n}$. 4, p. 571-590, 1966.

KRUGLIANSKAS, I. Engenharia simultânea e técnicas associadas em empresas tecnologicamente dinâmicas. Revista de Administração, v. 30, n. 2, p. 25-38, 1995.

MONTGOMERY, D. C. Diseño y análisis de experimentos. Trad. Jaime Delgado Saldivar. México: Iberoamérica, 1991.

MYERS, R. H.; MONTGOMERY, D. C. Response surface methodology: process and product optimization using designed experiments. Canada: John Wiley \& Sons, 1995.

STEINBERG, M. D.; HUNTER, W. G. Experimental design: review and comment. Technometrics, v. 26, n. 2, p. 71-130, May 1984.

TAGUCHI, G. Taguchi on robust technology development: bringing quality uspstream by Genichi Taguchi. New York: ASME, 1993.

O PLÁSTICO invade automóveis e caminhões. Gazeta Mercantil, São Paulo, 6 mar. 2002. Empresas \& Carreiras, p. $1-6$.

YONCHEV, H. New computer procedures for constructing Doptimal designs. In: DODGE, Y.; FEDOROV, V. V.; WYNN, H. P. Optimal design and analysis of experiments. New York: Elsevier Science Publishers B. V., 1988. p. 7195. 


\title{
APPLICATION DESIGN OF EXPERIMENTS IN THE INJECTION MOLDING PROCESS
}

\begin{abstract}
Industrial experiments are made by companies in order to improve the quality characteristics of products and production processes. In this sense, the objective of this paper is to study and apply the design of experiments in the industrial quality improvement. In addition, as a part of the objective, an application of the techniques of design Fractional Factorial $2^{k-p}$, Analysis of Variance and Response Surface Methodology is done. It is focused in an injection molding process applied by a company, that makes and trades plastic products for the civil construction. Using this experimental study, the most important parameters of plastic injection are identified: machine temperature and injection pressure. At the same time, the optimal levels of adjustment of these parameters are determined. From this study, it is evaluated both the implantation procedures of the designs of experiments as well as the difficulties faced. Also, this study tries to contribute to the university-company relationship.
\end{abstract}

Key words: industrial experiments, factorial fractional $2^{k-p}$, response surface methodology, injection-molding process. 